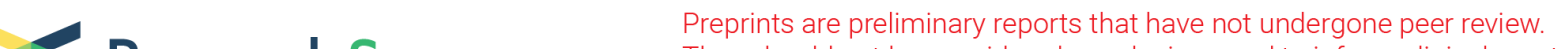 $\begin{array}{ll}\text { Research Square } & \text { They should not be considered conclusive, used to inform clinical practice, } \\ \text { or referenced by the media as validated information. }\end{array}$
}

\section{Postoperative Care of Two Patients With End-stage Heart Disease Combined With Type a Aortic Dissection}

\section{Xiaofeng Xu}

Guangdong Provincial Cardiovascular Institute, Guangdong Academy of Medical Sciences

\section{Yunqing Cheng}

Guangdong Provincial Cardiovascular Institute, Guangdong Academy of Medical Sciences

Juntao Kuang

Guangdong Provincial Cardiovascular Institute, Guangdong Academy of Medical Sciences

\section{Xuejun Xie}

Guangdong Provincial Cardiovascular Institute, Guangdong Academy of Medical Sciences

Jinsong Huang

Guangdong Provincial Cardiovascular Institute, Guangdong Academy of Medical Sciences

\section{Yanfen Wu}

Guangdong Provincial Cardiovascular Institute, Guangdong Academy of Medical Sciences

\section{Xueying Lan}

Guangdong Provincial Cardiovascular Institute, Guangdong Academy of Medical Sciences

Ruixin Fan ( $\nabla$ fanruixin@163.com )

Guangdong Provincial Cardiovascular Institute, Guangdong Academy of Medical Sciences

\section{Research Article}

Keywords: heart transplantation, acute type A aortic dissection, postoperative monitoring, nursing

Posted Date: April 26th, 2021

DOl: https://doi.org/10.21203/rs.3.rs-404805/v1

License: (c) (1) This work is licensed under a Creative Commons Attribution 4.0 International License. Read Full License 


\section{Abstract}

\section{Background}

Heart transplantation is a major method in the treatment of end-stage heart disease. Acute type A aortic dissection is a rare and life-threatening disease requiring emergency surgery. Although surgical treatment has achieved certain progress in terms of techniques and equipment in the recent years, the surgical mortality rate is still as high as $25 \%$. It is very rare for patients with end-stage heart disease to simultaneously contract aortic dissection. This study will explore the main points of care after end-stage heart disease combined with aortic dissection.

Results

The cold ischemic times of the donor heart of the two patients were $340 \mathrm{~min}$ and $361 \mathrm{~min}$, and the cardiopulmonary bypass times were $381 \mathrm{~min}$ and $411 \mathrm{~min}$. Both were successfully operated and discharged uneventfully.

\section{Conclusions}

The combination of heart transplantation with aortic dissection surgery is complicated and is prone to multiple postoperative complications. The nursing staff is required to master the main points of care after heart transplantation and aortic dissection, observe problems in a timely manner, intervene early, and promote the patient's recovery.

\section{Introduction}

Heart transplantation is an important approach in the treatment of end-stage heart disease and complex congenital heart disease [1]. Acute aortic dissection (AAD) is a rare life-threatening condition, and its diagnosis and treatment is still a challenge. According to the Stanford classification, AADs involving the ascending aorta are classified as type A dissections [2]. Acute type A AD (ATAAD) usually requires emergency surgery. Without surgery, $50 \%$ of patients would die within 24 hours of onset, and the mortality rate is about $1 \%-2 \%$ per hour [3]. Although we have made some progress in technology and equipment in recent years, the surgical mortality rate is still as high as $25 \%$ [4]. When surgery for these two diseases is performed at the same time, complications of various organ systems may occur postoperatively. Additionally, this combined operation has high requirements for postoperative medical management. Our center performed two heart transplants in combination with ATAAD, ascending aorta replacement, total aortic arch replacement, descending thoracic descending aortic stent elephant trunk surgery, in April 2018 and February 2019. In recent years, with the continuous improvement of the surgical technology in aortic dissection (AD), postoperative mortality has been significantly reduced, but early postoperative complications are still an independent risk factor for hospital mortality in patients with AD [5]. Additionally, infection is the main cause of death in patients after transplantation [6]. Postoperative medical treatments and nursing interventions are key to prevent and reduce complications, as well as an 
important guarantee for improving the efficacy of transplantation and improving the quality of life of the patients. This nursing report aims to explore the clinical nursing strategy for patients with end-stage heart disease and ATAAD.

\section{Methods}

\section{Clinical data}

Two patients were diagnosed with dilated cardiomyopathy and ATAAD. Case 1 (patient A), age, 62 years; height, $173 \mathrm{~cm}$; and weight, $56 \mathrm{~kg}$. On April 13, 2019, patient A was admitted to our hospital with "ATAAD, dilated cardiomyopathy, heart function grade IV." the patient had undergone percutaneous coronary intervention for coronary heart disease 7 years ago and had a favorable recovery. In the same year, patient A's transthoracic echocardiography (TTE) report showed dilated cardiomyopathy, severe mitral regurgitation, severe aortic regurgitation, and an ejection fraction (EF) of $65 \%$. Patient $A$ has received long-term treatments for high blood pressure, controlling heart rate, and antiplatelets. In October 2018, patient $A$ experienced shortness of breath after activity. The symptoms above worsened after half a year. Case 2 (patient B), age, 61 years; height, $160 \mathrm{~cm}$; and weight, $60 \mathrm{~kg}$. Patient B underwent mechanical aortic valve replacement in 1996 and mechanical mitral valve replacement in 2000. Patient B had a favorable recovery after the operation and took warfarin anticoagulant for a long time. In January 2019, patient B developed shortness of breath, chest tightness, and chest pain. After 3 months, patient B's TEE report revealed $A D$, severe tricuspid regurgitation, and moderate pulmonary hypertension. The systolic function of the left ventricle was significantly reduced, with an EF of $18 \%$.

\section{Surgical methods}

Both the patients underwent general anesthesia and deep hypothermia cardiopulmonary bypass for allogeneic orthotopic heart transplantation, ascending aorta replacement, total aortic arch replacement, descending thoracic descending aortic stent elephant trunk surgery at our center. After the donor heart was sutured, the nasopharyngeal temperature was reduced to $25^{\circ} \mathrm{C}$; whereafter, the circulation was arrested. The ascending aorta and the aortic arch were cut longitudinally, and the artificial vascular stent was inserted into the descending aorta. Island anastomosis technique was performed, and the arterial wall around the vessel island was reinforced. Surgeon trimmed the graft at the proximal end of the stent to create the vessel island and anastomose the stent vessel. Constructed the distal end of the ascending aorta for the donor heart to restore cardiac circulation and the heart to return to beating on its own.

\section{Surgical Result}

Both patients returned to the intensive care unit (ICU) easily after the operation. The ventilator assistance times of patient A was 106 hours and of patient B 130 hours. The ICU monitoring time of patient A was 8 days and of patient $B 24$ days, respectively. Early postoperative complications were one case each of renal insufficiency and chylothorax. No rejection had occurred. Both patients recovered and discharged. 


\section{Postoperative care}

ATAAD surgery and heart transplantation are both high-risk operations. In this study, two patients underwent the above two operations at the same time. These procedures are technically demanding. Although ATAAD surgery and heart transplantation has made technological advances in recent years, problems of large trauma, blood loss, severe postoperative conditions, and rapid changes still persist [7]. If effective monitoring, treatment, and care were not provided in time, the disease can progress and deteriorate at any time and may even lead to death. Therefore, nursing tasks are extremely substantial, and higher technical requirements are put forward for nursing.

\section{Hemodynamic monitoring}

Maintaining hemodynamic stability is the key to ensuring tissue perfusion. After transplantation, the donor heart suffers from different degrees of damage due to ischemia and reperfusion injury. Clinical manifestations such as increased central venous pressure (CVP), increased pulmonary artery pressure, hypotension, and oliguria will occur $[8,9]$. Therefore, postoperative inotropic drugs and vasodilators are routinely used to maintain cardiac function [10]. When multiple positive inotropic drugs are pumped intravenously, changes in the heart rate, heart rhythm, and blood pressure should be monitored, and the drug concentration and dose should be dynamically adjusted to avoid arrhythmia.

Patients in this study entered the laminar flow monitoring room after surgery and used a multi-function monitor to continuously monitor various hemodynamic indicators. The heart rate and rhythm, arterial blood pressure, mean arterial pressure, and CVP were recorded every $30 \mathrm{~min}$. Swan-Ganz floating catheters were placed after surgery to monitor cardiac output, pulmonary artery pressure, and pulmonary capillary wedge pressure. At postoperative 24 hours, the above indicators were recorded at every hour, and the cardiac output index was monitored every 8 hours to guide treatment and nursing measures. The pericardial mediastinal drainage and urine output was recorded every hour and the peripheral circulation of the limbs was also monitored. The patient's heart rate was maintained at $90-110$ beats $/ \mathrm{min}$ and the blood pressure at 100-120/60-70 $\mathrm{mmHg}$.

When a special medicine was used, it was pumped at a constant rate through a micro pump. The effect of the medicine was closely observed. Both cases received intravenous epinephrine $0.025-0.08 \mu \mathrm{g} / \mathrm{kg} \cdot \mathrm{min}$ after surgery. One patient was administered norepinephrine $0.025-0.20 \mu \mathrm{g} / \mathrm{kg} \cdot \mathrm{min}$ and levosimendan 0.1 $\mu \mathrm{g} / \mathrm{kg} \cdot \mathrm{min}$ because the blood pressure was as low as $80 / 30 \mathrm{mmHg} 24$ hours after surgery. In the other case, epinephrine was stopped 24 hours after surgery and replaced with dopamine $5 \mu \mathrm{g} / \mathrm{kg} \cdot \mathrm{min}$ and levosimendan $0.1 \mu \mathrm{g} / \mathrm{kg} \cdot \mathrm{min}$. The arterial blood pressure and mean arterial pressure were maintained at 98-120/55-85 mm Hg and $>60 \mathrm{~mm} \mathrm{Hg}$. The dosage was adjusted according to the patient's clinical efficacy. Moreover, the continuous and accurate administration of the medicine was ensured to maintain the stability of the circulation.

\section{Strictly manage the amount of input and output to ensure the balance of water and electrolyte}


CVP refers to the blood pressure near the right atrium, superior vena cava, or inferior vena cava. It is an important indicator for evaluating blood volume and right heart function [11]. CVP was monitored Q1h after surgery, and at Q2h was changed the next day. CVP should be maintained below $10 \mathrm{~cm} \mathrm{H}_{2} \mathrm{O}$, while maintaining a stable blood pressure [12]. In the early stage, due to the large amount of water in the body, diuresis should be enhanced, and colloidal fluid should be infused to ensure normal cardiac output [13, 14], while maintaining good hemodynamics to prevent hypotension. When AD was complicated by heart failure, or when the dissection involved the renal artery, it can cause renal malperfusion. Additionally, improper perioperative antihypertensive treatment can cause hypotension and lead to poor renal perfusion. Tissue ischemia caused by intraoperative circulation arrest and reperfusion injury caused by cardiopulmonary bypass can lead to renal damage [10]. Cardiopulmonary bypass surgery will present as low on the urinometer [15]. Therefore, we noted the amount of input and output every day for 24 hours, and kept the balance of the amount of input and output.

The CVP of the two patients was maintained between $11-15 \mathrm{~cm} \mathrm{H}_{2} \mathrm{O}$ on the day after surgery and rose to $19-20 \mathrm{~cm} \mathrm{H}_{2} \mathrm{O}$ on the $3^{\text {rd }}$ and $4^{\text {th }}$ day after surgery, respectively. The nurse conveyed to the doctor to perform diuresis in time and controlled the amount and rate of infusion to maintain CVP below $13 \mathrm{~cm}$ $\mathrm{H}_{2} \mathrm{O}$. The CVP of the two patients was maintained below $10 \mathrm{~cm} \mathrm{H}_{2} \mathrm{O}$ on the $7^{\text {th }}$ and $8^{\text {th }}$ day after operation. Troponin T, troponin I, and pro brain natriuretic peptide were tested before and three days after surgery. The nurse maintained electrolyte and acid-base balance, and gave special attention to preventing hypokalemia, hyperkalemia, and acidosis. One of the patients had low urine output after surgery and was given hemodialysis. The hemodialysis process accurately recorded the dialysis time, amount of dehydration, and amount of heparin. Patient's femoral venous hemodialysis tube should be observed during each shift for any bleeding, exudate, etc. In addition, patients were instructed not to compress the tube placement when moving or turning to prevent the tube from falling off.

\section{Arrhythmia monitoring}

After heart transplantation, the donor's heart loses its innervation, which leads to poor neuroregulation and body fluid regulation. Therefore, postoperative arrhythmia can often occur. Echahidi's study [16] demonstrated that the incidence of atrial fibrillation after cardiac surgery was relatively high, with a total incidence of nearly $40 \%$.

After entering the ICU, the patient's heart rate and heart rhythm changes were closely monitored. The bedside 12-lead electrocardiogram was checked once a day for a week. In both cases, temporary pacemakers were indwelled during the operation. Nursing work included properly fixing the pacing lead, observing the pacing effect, and battery signal, and timely adjustment of the pacemaker parameters. We adjusted the pacing heart rate to $80-100$ beats/min, the sensitivity was $1.6-2.0 \mathrm{mv}$, and the output voltage was 2.0-6.0 V. During the early recovery of the spontaneous heart rate, the pacemaker and pacing frequency were kept slower than the spontaneous heart rate. Two patients in this group had sinus rhythm after operation, and no complicated ventricular arrhythmia or atrioventricular separation occurred. 


\section{Respiratory function support}

\section{Mechanical Ventilation}

Patients with heart transplantation combined with aortic dissection have poor cardiopulmonary function and unstable disease. To ensure oxygen supply and maintain normal gas exchange after the operation, both patients were returned to the ICU and were placed on a ventilator to assist breathing. According to the blood gas test results and oxygenation index in the early postoperative period, the ventilator parameters should be adjusted reasonably. If $\mathrm{PaO}_{2} / \mathrm{FiO}_{2} \leq 200 \mathrm{~mm} \mathrm{Hg}$, a high-frequency low-tidal volume lung protective ventilation strategy was adopted. This strategy could limit the excessive expansion of the alveoli in the non-gravity dependent area at the end of expiration and maintain the optimal lung volume at the end of expiration. This was conducive to the expansion of collapsed alveoli during the operation, increasing the ratio of ventilation to blood flow and the oxygenation index [17]. In patients with hypoxemia, lung compliance decreases and airway resistance increases. The airway platform pressure should be maintained below $30 \mathrm{~cm} \mathrm{H} \mathrm{H}_{2} \mathrm{O}$. Patients usually need to be sedated, and muscle relaxants should be given if necessary [18].

The ventilator modes used by the patients in this study were all synchronized intermittent mandatory ventilation (SIMV). The parameter settings in this mode were: $\mathrm{FiO}_{2} 80 \%, \mathrm{VT} 450 \mathrm{ml}(<8 \mathrm{ml} / \mathrm{kg})$, positive end-expiratory pressure $8 \mathrm{~cm} \mathrm{H}_{2} \mathrm{O}, \mathrm{F} 16$ times/min, $\mathrm{E}: \mathrm{I}=1$ : 1.5 . The patient's spontaneous breathing rate and rhythm were closely observed by the nurse, and changes in blood pressure and heart rate were also monitored. The peripheral blood oxygen saturation should be closely monitored and blood gas test should be performed conditionally. Depending on the blood gas results, the nurse contacted the doctor in time to adjust the ventilator parameters. The nurse must ensure the normal operation of the ventilator and detect the various alarms in time.

\section{Prevention of hypoxemia}

The hypothermia and reduction of lung tissue perfusion caused by deep hypothermic circulatory arrest, as well as systemic inflammatory response caused by ATAAD, all increased the incidence of acute lung injury. The symptoms of lung injury in most patients can be improved after perioperative lung protection treatment, but a considerable number of patients who experienced aggravation and eventually died were still known [19]. Patients with acute lung injury requires to maintain artificial airway and mechanical ventilation, which leads to prolonged ICU time and average hospital stay. Therefore, it is extremely important to treat postoperative hypoxemia.

The two patients in this study were not fully awake and restless after the operation. They were ventilated using the ventilator, which caused the airway pressure of the ventilator to rise, and the symptoms of hypoxia appeared. In this condition, dexmedetomidine $1 \mathrm{mg} / \mathrm{kg} / \mathrm{h}$ was used with micropump in accordance with the doctor's advice. The sputum suction at our center was used with a closed suction tube, which would not interrupt the oxygen supply, and at the same time avoided cross-infection caused by sputum ejection. One patient developed low blood oxygen partial pressure on the third day after 
surgery, and the traditional sputum suction effect was poor. Therefore, bronchoscopy was performed for sputum suction, which significantly improved the patient's blood gas status and lung function. Another patient developed tridentation on the $4^{\text {th }}$ day after the tracheal intubation was removed, and the blood oxygen saturation decreased progressively. The tracheal intubation was immediately reintubated. After the improvement of blood oxygen saturation, he changed to double nasal canal oxygen on the $14^{\text {th }}$ day after operation.

\section{Prevention of infection}

Infection is the main complication after a transplantation [20], with an annual fatality rate of nearly $20 \%$, which is the main cause of death in transplant patients [6]. Among the many causes, opportunistic infections caused by the application of immunosuppressants are the most prominent. Additionally, infection is also a common complication after ATAAD. Previous studies have shown that independent risk factors for infection within 6 months after surgery include advanced age, ventilator support, ventricular assist devices, OKT3 induction therapy. [21].

One patient in this study had a small amount of exudation from both the lungs on the bedside chest radiograph on the second day after surgery, and the blood culture indicated Klebsiella. The nursing measures of our center were $\nabla$ strict aseptic operation: nurse maintained aseptic operation when invasive operations were performed on the patient, such as sputum suction, puncture and intubation, blood transfusion; $₫$ protective quarantine: direct entry of the patient into the sterile single room from the operating room; in addition to reduced personnel access and special care. All items in the room were disinfected with ultraviolet rays or were wiped with chlorine-containing disinfectant before entering. Temperature and humidity indoors was maintained properly. In case of family visits, relevant missions were provided and protective isolation must be conducted. $\otimes$ Antibiotics and antiviral drugs should be prophylactically used. $\otimes$ Oral care: If patients had oral ulcers, they should be given regular oral care, and nystatin must be applied to the ulcer surface, 3-4 times a day. Nurse checked the patients' oral leukoplakia or ulcers during each shift and the patients were instructed to rinse their mouths before and after meals. $\otimes$ Pulmonary care: under the guidance of a cardiac rehabilitation specialist, nurse would turn the patient over and pat their back to prevent falling pneumonia. Inhalation drug therapy was performed four times a day to prevent lung infection; $₫$ Prevention of skin complications: The skin of the patient was wiped every morning and evening after the operation. $\otimes$ The indwelling pipesuch as a thoracic and pericardial drainage tube, central venous catheter, and urinary catheter, must be removed as soon as possible.

\section{The application of immunosuppressive agents and the observation of rejection}

Acute immunological rejection usually occurs early on after transplantation and is easier to detect. The application of a variety of new anti-rejection drugs has made acute rejection easier to control. Chronic rejection occurs in the late stage after transplantation. Effective treatment drugs for rejection are lacking, so the treatment is very difficult. Studies have shown that the graft dysfunction could reach $50 \%$ after 3 
years of heart transplantation [22]. Strictly observed acute rejection after operation include fever, unexplained drop in blood pressure. At the same time, observation and education of adverse reactions of immunosuppressants should be considered. After taking immunosuppressive agents, patients may experience different degrees of gastrointestinal reactions, bone marrow suppression, and etc. Patients would present with nausea and vomiting, leukopenia, and etc. Additionally, hormones can cause hair to increase and thicken. Patients were instructed not to discontinue the drug without consultation because of the changes in appearance.

The timely and reasonable application of immunosuppressants and close monitoring after heart transplantation can effectively reduce the occurrence of rejection. Two patients in this study were given basiliximab $20 \mathrm{mg}$ and methylprednisolone $500 \mathrm{mg}$ intravenously. In the first 3 days after the operation, both cases received methylprednisolone $500 \mathrm{mg}$ intravenous infusion of pulse therapy once a day. After operation, FK506, mycophenolate mofetil capsules, and methylprednisolone were used to prevent immunosuppression and treat rejection. The blood concentration of the immunosuppressant tacrolimus was regularly monitored and maintained at $20-25 \mathrm{ng} / \mathrm{mL}$. Before discharge, patients and their families were instructed to identify signs of rejection and issue education sheets. Patients were informed to take immunosuppressive agents as prescribed by the doctor for life, and to go to the hospital for regular review. The patients were discharged for 6 months and visited the hospital for follow-up. No rejection had occurred in the two patients.

\section{Nervous system care}

Nervous system complications are the most common complications after cardiac surgery, transient brain dysfunction accounts for the majority, followed by spinal cord injury and cerebral infarction [23]. A Japanese study demonstrated that deep hypothermia itself and long brain perfusion time would aggravate nerve damage, which was more obvious in the elderly patients [24]. Both patients in this study were awake 4 days after the surgery. One patient developed mental symptoms, manifested as cognitive impairment, hallucinations, delirium, and restlessness. He was treated with Xingnaojing injection, methylprednisolone, and olanzapine, and the patient's mental state gradually recovered. Previous study showed that preoperative hypertension, especially refractory hypertension with poor drug control, was a risk factor for neurological complications after surgery [25]. Both patients had a history of hypertension before surgery, and their arterial blood pressure was closely monitored after surgery. In this study, we controlled the patient's systolic blood pressure at 100-120 mm Hg. And we determined the GLASS scores, measured the pupil size, and checked the light reflection at every 4 hours after the patient had entered the ICU. A nurse observed the activity of the limbs every 8 hours and conducted muscle strength assessment. Additionally, we monitored the patient's limb arterial pulsation, skin color, temperature, and pain response. Neither of the two patients in this group presented with paraplegia or limb weakness.

\section{Pipe care}

Both cases were intubated with one each of a thoracic and pericardial drainage tube, central venous catheter, and urinary catheter. Nursing work included: observing the nature and quantity of the drainage 
fluid and the temperature of the drainage fluid. When the drainage volume lasted for $2 \mathrm{~h}>200 \mathrm{~mL}$, or when the drainage fluid was red and warm, it was indicative of active bleeding. Whereafter, it was necessary to get ready for bedside thoracotomy. If the pericardial drainage tube suddenly decreased, acute cardiac tamponade should be prevented, which can clinically manifest as a decrease in blood pressure, an increase in the heart rate, pallor, profuse sweating, and jugular venous dilation. Therefore, a nurse would check the scale of each pipeline at every shift, change the dressing in time, and squeeze the chest tube regularly to prevent blood clots from blocking the pipeline. Each pipeline was wrapped with a sterile treatment towel to ensure sterility. Patient $A$ had an increase in the pleural fluid volume on the $4^{\text {th }}$ day after the operation, and the highest peak of $1530 \mathrm{ml}$ appeared on the $10^{\text {th }}$ day. The chyle test was positive. Fat-free and high-protein foods were provided according to the advice of the nutrition department to maintain their nutritional status. The chest tube was removed 28 days after the operation, the chyle test was negative, and the nutritional score (NRS2002) was 2 points. In the other patient, the thoracic cavity and pericardial drainage tube were removed 13 days after the operation.

\section{Conclusion}

Two patients with heart transplantation combined with AD were discharged from the hospital after strict observation and care of their condition. The patient had no cardiac insufficiency or rejection in the 6month follow-up after discharge. Heart transplantation combined with $A D$ is prone to various serious complications after the operation, involving various tracheal systems. Therefore, nursing staff should understand the clinical manifestations of the various complications, which is conducive to their early detection and prevention. The application of immunosuppressive agents should emphasize individualization, depending on the recipient's immune status and systemic organ function status. Therefore, postoperative discharge education and regular follow-up of patients are particularly important. At present, reports of patients with such a surgery are few, and the medical care experience is limited. Therefore, nursing experience and long-term treatment and nursing strategies should be explored further.

\section{Declarations}

\section{Funding}

Supported by National Key Research and Development Program of China (2017YFC1308003). The funding body supported collection, professional advice from a statistician in Southern Medical University for suitable statistical analyses and from Wiley Editing Service for the quality of English. All the authors have nothing to disclose.

\section{Ethics approval and consent to participate}

This retrospective study was approved by the ethics committee of Guangdong Provincial People's Hospital, Guangdong Academy of Medical Sciences, Guangzhou, China (No.GDREC2018322H(R2)). 
Written and informed consent was obtained from the patients included in this study. All methods were performed in accordance with the relevant guidelines and regulations.

\section{Authors' contributions}

XXF and CYQ, contributions to conceptualization and design, or acquisition of data, or interpretation of data, involved in drafting, reviewing and editing the manuscript; KJT, contributions to acquisition of data, involved in reviewing and editing the manuscript; XXJ, HJS, WYF and LXY, interpretation of data, involved in reviewing and editing the manuscript; FRX, revising it critically for important intellectual content. All authors read and approved the final manuscript.

\section{Consent for publication}

Not applicable.

\section{Competing interests}

Not applicable.

\section{Availability of data and materials}

All data generated or analysed during this study are included in this published article.

\section{Acknowledgements}

Not applicable.

\section{References}

[1]Dipchand Al, Kirk R, Edwards LB, Kucheryavaya AY, Benden C, Christie JD, et al. The Registry of the International Society for Heart and Lung Transplantation: Sixteenth Official Pediatric Heart Transplantation Report-2013; Focus Theme: Age. J Heart Lung Transplant, 2013, 32 (10): 979-988.

[2]LeMaire SA, Russell L. Epidemiology of thoracic aortic dissection. Nat Rev Cardiol 2011;8(2):103-113.

[3]Hagan PG, Nienaber CA, Isselbacher EM, Bruckman D, Karavite DJ, Russman PL, et al. The International Registry of Acute Aortic Dissection (IRAD): new insights into an old disease. JAMA, 2000, 283: 897-903.

[4]Mehta RH, Suzuki T, Hagan PG, Bossone E, Gilon D, Llovet A,et al. Predicting death in patients with acute type A aortic dissection. Circulation, 2002 (105): 200-206.

[5]Oderich GS, Panneton JM, Bower TC, et al『Aortic dissectionwith aortic side branch compromise: impact of malperfusion on patient outcome区Perspect Vasc Surg Endovasc Ther, 2008, 20: 190-200区 
[6]Sharkey A, Warriner D, Braidley P. Surgical management of endstage heart failure. Br J Hosp Med ( Lond), 2012, 73 (11): 633-639.

[7]Chen LW, Dai XF, Lu L, et al. Extensive primary repair of the thoracic aorta in acute type A aortic dissection by means of ascending aorta repiacement combined with open placement of triple-branched stent graft early results. Circulation, 2010, 122 (14): 1373-1378.

[8]Shi L, Huo CY, Liu JL, et al. IABP combined with ECMO assisted treatment of patients with severe low cardiac output syndrome after cardiac surgery. Chin J Modern Nurs, 2013, 19 (13): 1531-1533.

[9]Guo JQ, Wu QYヌ2003, p: 886. Nursing care in cardiac surgery \China, Beijing: people's medical publishing house $₫ 963 \mathrm{pp} \rrbracket$

[10]Chen XF, Wang DJ, Xu B, et al. Effects of hypertension on the early central nervous system function in patients after surgery for aortic dissection囚Chin J Hypertens, 2012, 20 (2): 171-174区

[11]Miryam MR, Marcel A囚Central venous pressure: principles, measurement, and interpretation $\bigotimes$ Compend Contin Educ Vet. 2012 Jan; 34 (1): E1.

[12]Gary SF, Barry HG, Daphne TH, et al. ACCF/AHA/ACP/HFSA/ISHLT 2010 Clinical Competence Statement on Management of Patients With Advanced Heart Failure and Cardiac Transplant: A Report of the ACCF/AHA/ACP Task Force on Clinical Competence and Training. 56 (5): 424-453.

[13]Shi L, Huo CY, Liu JL, et al. Observation and Nursing of Complications of Mechanical Auxiliary Treatment after Cardiac Operation. Chin J Modern Nurs, 2019 (14): 1684-1686.

[14]Wei YY, Shi L, Wu R. Nursing Care of 8 Patients with Left Heart Assisted Circulation Device. Chin J Nurs, 2011, 046 (11): 1134-1135.

[15] Xu HY. Perioperative nursing care of 5 patients with Stanford type A aortic dissection. South China J Cardiovasc Diseases, 2013, 19, (4): 518-520

[16] Echahidi N, Pibarot P, O'Hara G, et al囚Mechanisms, prevention, and treatment of atrial fibrillation after

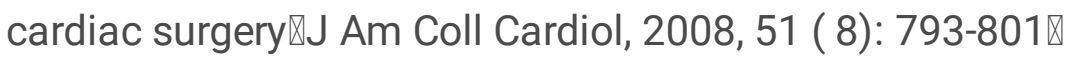

[17] Michael CS, Eddy F, Ewan G『High-Frequency Oscillatory Ventilation in Adults With ARDS®Chest, 2017, 6 (25): 1306-1317区

[18]Bein T, Grasso S, Moerer O, et alषThe standard of care of patients with A\&DS: ventilatory settings and rescue therapies for refractory hypoxemia冈Intensive Care Med, 2016, 42 (5): 699-711》

[19]Evaldas FD, Thomas TC, Micheal HS, et al. Acute respiratory dysfunction after surgery for acute type A aortic dissection. Eur J Cardiothorac Surg, 2010, 37: 691-696. 
[20]Kijpittayarit-Arthurs S, Eid AJ, Kremers WK, et al. Clinical features and outcomes of delayed-onset primary cytomegalovirus disease in cardiac transplant recipients. J Heart Lung Transplant, 2007, 26 (10): 1019-1024.

[21]Smart FW, Naftel DC, Costanzo MR, et al. Risk factors for early, cumulative, and fatal infections after heart transplantation : a multiinstitutional study. J Heart Lung Transplant, 1996, 15 (4): 329-341.

[22] Lattmann T, Hein M, Horber S, et al. Activation of pro-inflammatory and anti-inflammatory cytokines in host organs during chronic allograft rejection: role of endothelin receptor signaling. Am J Transplant . 2005 May; 5 (5): 1042-9.

[23]Hunaid AV, Wadih RT, Dimitri SE $\ T$ Temporary neurological dysfuction after surgery of the thoracic aorta: a predictor of poor outcome and impaired quality of life区 Eur J Cardiothorac Surg, 2008, 33: 10251029凶

[24] Hata M, Sezai A, Yoshitake I, et al囚Clinical trends in optimal treatment strategy for type A acute aortic dissection囚Ann Thorac Cardiovasc Surg, 2010, 16: 228-235区

[25]Liu DW. 2010, p: 110-113. Critical Care Medicine. China, Beijing: people's medical publishing house区 $413 \mathrm{pp}$. 